\title{
Assessment of autonomic nervous system function in nursing students using an autonomic reflex orthostatic test by heart rate spectral analysis
}

\author{
MAO HASEGAWA $^{1}$, AZUSA HAYANO ${ }^{1}$, ATSUSHI KAWAGUCHI ${ }^{2}$ and RYUYA YAMANAKA ${ }^{1}$ \\ ${ }^{1}$ Department of Medical Science, Graduate School of Nursing for Health Care Science, Kyoto Prefectural University \\ of Medicine, Kyoto 602-8566; ${ }^{2}$ Department of Biomedical Statistics and Bioinformatics, \\ Graduate School of Medicine, Kyoto University, Kyoto 606-8501, Japan
}

Received June 29, 2015; Accepted August 4, 2015

DOI: 10.3892/br.2015.512

\begin{abstract}
Nursing students experience academic demands, such as tests, theoretical and practical coursework, research activities, various aspects of professional practice, and contact with health professionals and patients. Consequently, nursing students face numerous types of stress, and increased stress levels contribute to physical and psychological distress in nursing students. The aim of the present study was to investigate the autonomic nervous system function of nursing students by assessing active standing load using the autonomic reflex orthostatic tolerance test, which enables quantitative analysis of dynamic autonomic nervous system function. The autonomic nervous system activity in the resting state was low in fourth-year students, they had parasympathetic hypotension, and there was a tendency towards higher sympathetic nervous system activity of fourth-year students compared with first-, second- and third-year students. In the standing state, there was a trend towards a higher autonomic nervous system activity response of fourth-year students compared with first-, second- and third-year students. These results suggest that stress may influence autonomic nervous activity in fourth-year nursing students. By correcting stress in fourth-year nursing students, it may be possible to prevent the development of health problems.
\end{abstract}

\section{Introduction}

The autonomic nervous system coordinates bodily functions to ensure homeostasis (cardiovascular and respiratory control, thermal regulation, gastrointestinal motility, urinary and bowel

Correspondence to: Dr Ryuya Yamanaka, Department of Medical Science, Graduate School of Nursing for Health Care Science, Kyoto Prefectural University of Medicine, 465 Kajii-cho, Kyoto 602-8566, Japan

E-mail: ryaman@koto.kpu-m.ac.jp

Key words: autonomic nervous activity, heart rate variability analysis, autonomic reflex orthostatic tolerance test, nursing school students, stress reaction excretory functions, reproduction, and metabolic and endocrine physiology) and adaptive responses to stress (1). Autonomic responses to psychological stress induce activation of sympathetic nerves with inhibition of the parasympathetic nervous system and adrenocortical hormone release to the heart, leading to increased heart rate, cardiac output and blood pressure (2). Psychological factors contribute to physiological processes (such as sweating, cardiovascular and gastrointestinal arousal) via the autonomic nervous system (3). Stress is a major contributor to psychosocial and physical pathological conditions (4).

Nursing students experience academic demands, such as tests, theoretical and practical coursework, research activities, various aspects of professional practice, and contact with health professionals and patients. Consequently, nursing students face numerous types of stress (5). Thus, increased stress levels contribute to physical and psychological distress in nursing students (6-8). There is a concern that the added demands of modern nursing programs place the students under greater pressure due to competing demands (9). Therefore, we hypothesized that high-grade students faced greater stressors, and consequently had abnormal functioning of the autonomic nervous system.

The aim of the present study was to examine the functioning of the autonomic nervous system in nursing students by assessing active standing load using the autonomic reflex orthostatic tolerance test that enables quantitative analysis of dynamic autonomic nervous system function.

\section{Materials and methods}

Design and participants. There were 121 nursing students (22 first-, 35 second-, 23 third- and 41 fourth-year students) enrolled at the Kyoto Prefectural University of Medicine (Kyoto, Japan). These data are $>70 \%$ of stable R-R intervals without noise. There were $3(2.5 \%)$ males and $118(97.5 \%)$ females, and the average age was $20.6 \pm 1.8$ years (range, 18-33 years). The investigation was carried out between October 1, 2014 and January 31, 2015.

Data collection and analysis. For measurements of the autonomic nervous system functions, a heart rate variability 
Table I. Autonomic nervous system functions of the resting state in different student nursing years.

\begin{tabular}{lccccc}
\hline Variables & $\begin{array}{c}\text { First year, } \\
\mathrm{n}=22\end{array}$ & $\begin{array}{c}\text { Second year, } \\
\mathrm{n}=35\end{array}$ & $\begin{array}{c}\text { Third year, } \\
\mathrm{n}=23\end{array}$ & $\begin{array}{c}\text { Fourth year, } \\
\mathrm{n}=41\end{array}$ & $\begin{array}{c}\text { Wilcoxon/Kruskal-Wallis } \\
\text { testing result }\end{array}$ \\
\hline CVRR & $5.80 \pm 1.53$ & $5.50 \pm 1.55$ & $5.68 \pm 1.75$ & $5.02 \pm 1.37$ & First vs. fourth; P=0.017 \\
LF/HF & $2.97 \pm 2.34$ & $2.80 \pm 2.44$ & $2.10 \pm 1.97$ & $3.69 \pm 3.57$ & NS \\
CCVHF & $2.46 \pm 0.75$ & $2.36 \pm 0.77$ & $2.73 \pm 0.89$ & $2.23 \pm 0.85$ & Third vs. fourth; P=0.015 \\
Heart rate & $73.72 \pm 9.50$ & $70.82 \pm 8.90$ & $72.70 \pm 11.42$ & $73.18 \pm 8.98$ & NS \\
\hline
\end{tabular}

Data are average \pm standard deviation. NS, not significant; CVRR, coefficient of variation of R-R intervals; LF, low-frequency; HF, high-frequency; CCVHF, coefficient of component variance for HF.

Table II. Autonomic nervous system functions of the standing state in different student nursing years.

\begin{tabular}{lccccc}
\hline Variables & $\begin{array}{c}\text { First year, } \\
\mathrm{n}=22\end{array}$ & $\begin{array}{c}\text { Second year, } \\
\mathrm{n}=35\end{array}$ & $\begin{array}{c}\text { Third year, } \\
\mathrm{n}=23\end{array}$ & $\begin{array}{c}\text { Fourth year, } \\
\mathrm{n}=41\end{array}$ & $\begin{array}{c}\text { Wilcoxon/Kruskal-Wallis } \\
\text { testing result }\end{array}$ \\
\hline$\Delta \mathrm{CVRR}$ & $2.57 \pm 2.13$ & $2.38 \pm 2.56$ & $2.76 \pm 1.71$ & $3.35 \pm 2.09$ & $\mathrm{NS}$ \\
$\Delta \mathrm{LF} / \mathrm{HF}$ & $4.20 \pm 7.47$ & $3.94 \pm 7.88$ & $3.60 \pm 4.39$ & $2.60 \pm 3.61$ & $\mathrm{NS}$ \\
$\Delta \mathrm{CCVHF}$ & $0.57 \pm 0.41$ & $0.46 \pm 0.40$ & $0.48 \pm 0.65$ & $0.38 \pm 0.71$ & $\mathrm{NS}$ \\
Heart rate & $7.55 \pm 4.27$ & $7.47 \pm 4.43$ & $7.48 \pm 3.45$ & $8.03 \pm 4.53$ & $\mathrm{NS}$ \\
\hline
\end{tabular}

Data are average \pm standard deviation. NS, not significant; CVRR, coefficient of variation of R-R intervals; LF, low-frequency; HF, high-frequency; CCVHF, coefficient of component variance for HF.

analysis method was used (10). Heart rate and autonomic nervous activity were measured. Electrocardiogram measurements were obtained in a sitting position for $2 \mathrm{~min}$, in a standing position for $2 \mathrm{~min}$ and in a resting position for $1 \mathrm{~min}$ for autonomic reflex orthostatic tolerance test. Real-time analysis was performed using heart rate variability analysis software by Crosswell Co., Inc., (Yokohama, Japan). The coefficient of variation of R-R intervals (CVRR) obtained from electrocardiograms were divided into high-frequency (HF) and low-frequency (LF) components by power spectrum analysis to evaluate the autonomic nervous activity. The HF component reflects parasympathetic nervous activity, while the LF/HF reflects sympathetic nervous activity. Coefficient of component variance for HF (CCVHF) reflects the parasympathetic nervous index, and CVRR as the variability of overall autonomic nervous system activity. $\triangle \mathrm{CVRR}$ was used as the response of the autonomic nervous system. $\Delta \mathrm{LF} / \mathrm{HF}$ was used as the sympathetic nervous system function in the standing position. $\triangle \mathrm{CCVHF}$ was used as the parasympathetic nervous system function in the sitting position. The differences between the groups of each parameter were analyzed using the Wilcoxon/Kruskal-Wallis test. A two-tailed $\mathrm{P}<0.05$ was considered to indicate a statistically significant difference.

Ethics consideration. The study received Ethics approval from the Institutional Review Board of the Kyoto Prefectural University of Medicine (notice no. of determination: ERB-E-76, 2014). The students received an information sheet that explained the purpose of the study and methods, and were provided with assurance that participation was voluntary and that their personal information would be kept confidential during and following the study.

\section{Results}

Resting and standing states. The results of every student year for resting state are shown in Table I. The activity of the autonomic nervous system (CVRR) of fourth-year students was lower compared with first-year students (fourth-year

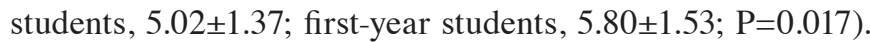
There was a trend of the sympathetic nervous system activity (LF/HF) of fourth-year students to be higher compared with other student years (fourth-year students, 3.69 \pm 3.57 ; third-year students, $2.10 \pm 1.97 ; \mathrm{P}=0.08)$. The parasympathetic nervous system activity (CCVHF) of fourth-year students was lower compared with third-year students (fourth-year students, 2.23 \pm 0.85 ; third-year students, 2.73 \pm 0.89 ; $\mathrm{P}=0.015$ ). The results for every student years for the standing state are shown in Table II. There was a trend of $\triangle$ CVRR of fourth-year students to be higher compared with other student years (fourth-year students, 3.35 \pm 2.09 ; second-year students, $2.38 \pm 2.56 ; \mathrm{P}=0.10)$. There was a trend of the sympathetic nervous system response $(\Delta \mathrm{LF} / \mathrm{HF})$ of fourth-year students to be lower among the other student years.

Autonomic nervous system functions. The results of the autonomic nervous system functions of the resting state comparing fourth-year students with first- to third-year students is shown in Fig. 1. The activity of the autonomic nervous system (CVRR) of fourth-year students was lower compared with first- to third-year students (fourth-year students, 5.02 \pm 1.37 ; 
A

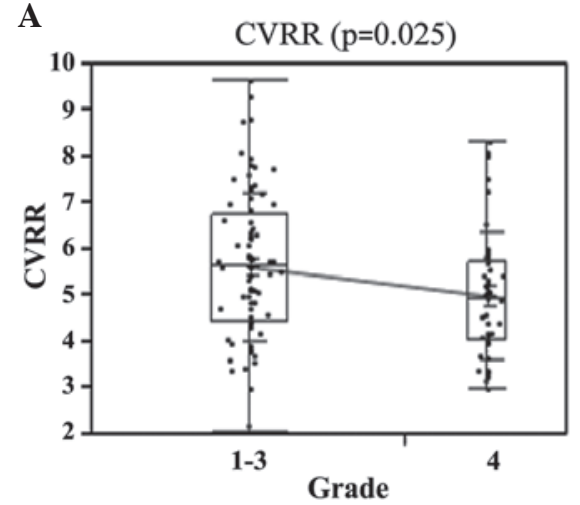

B

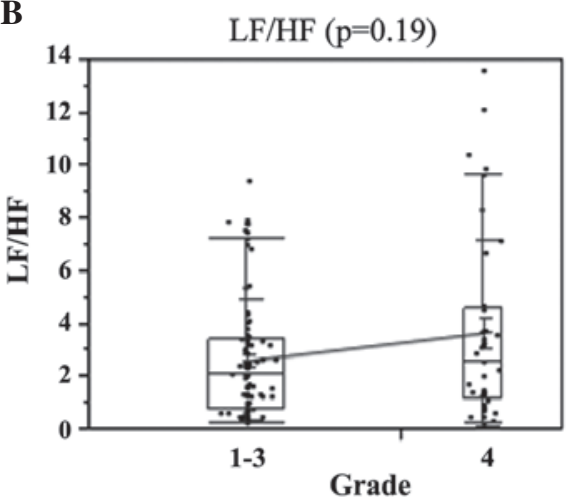



Figure 1. Autonomic nervous system functions at the resting state comparing fourth-year nursing students with first- to third-year nursing students. (A) CVRR $(\mathrm{P}=0.025),(\mathrm{B}) \mathrm{LF} / \mathrm{HF}(\mathrm{P}=0.19)$ and $(\mathrm{C}) \mathrm{CCVHF}(\mathrm{P}=0.04)$. CVRR, coefficient of variation of R-R intervals; LF, low-frequency; HF, high-frequency; CCVHF, coefficient of component variance for $\mathrm{HF}$.

A

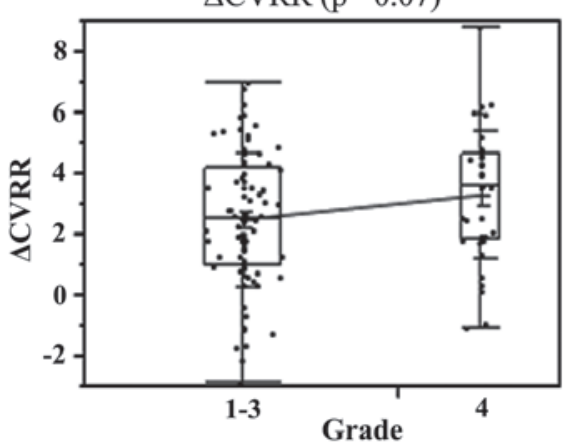

B

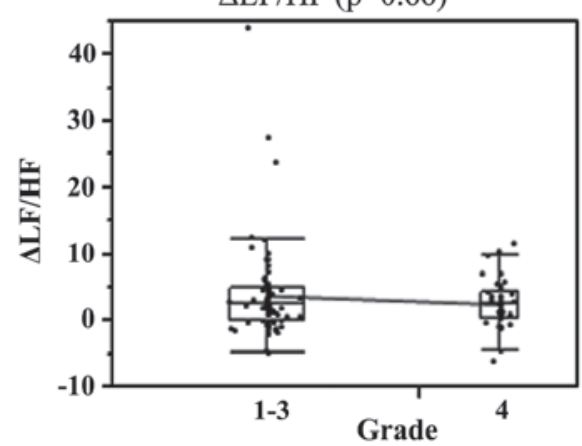

C

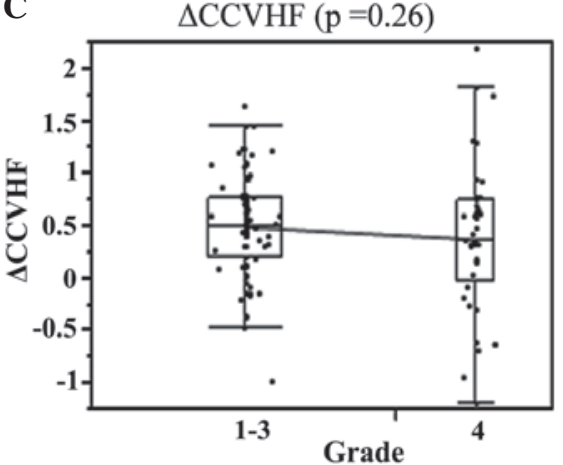

Figure 2. Autonomic nervous system functions at the standing state comparing fourth-year nursing students with first- to third-year nursing students. (A) $\triangle$ CVRR $(\mathrm{P}=0.07),(\mathrm{B}) \triangle \mathrm{LF} / \mathrm{HF}(\mathrm{P}=0.66)$ and $(\mathrm{C}) \triangle \mathrm{CCVHF}(\mathrm{P}=0.26)$. CVRR, coefficient of variation of R-R intervals; LF, low-frequency; HF, high-frequency; CCVHF, coefficient of component variance for $\mathrm{HF}$.

first- to third-year students, $5.63 \pm 1.59 ; \mathrm{P}=0.025)$. There was a trend of the sympathetic nervous system activity (LF/HF) of fourth-year students to be higher compared with first- to third-year students (fourth-year students, 3.69 \pm 3.57 ; first- to third-year students, 2.65 \pm 2.29 ; $\mathrm{P}=0.19)$. The parasympathetic nervous system activity (CCVHF) of fourth-year students was lower compared with first- to third-year students (fourth-year students, 2.23 \pm 0.85 ; first- to third-year students, $2.49 \pm 0.81$; $\mathrm{P}=0.04)$. The results of the autonomic nervous system function in the standing state comparing fourth- to first- to third-year students is shown in Fig. 2. There was a trend of the $\triangle$ CVRR of the fourth-year students to be higher compared with first- to third-year students (fourth-year students, 3.35 \pm 2.09 ; first- to third-year students, $2.54 \pm 2.20 ; \mathrm{P}=0.07) . \Delta \mathrm{LF} / \mathrm{HF}$ and $\triangle \mathrm{CCVHF}$ were not significant between fourth- and first- to third-year students.

\section{Discussion}

The present study showed that fourth-year student autonomic nervous system activity in the resting state was low, there was parasympathetic hypotension and there was a trend of the sympathetic nervous system activity of fourth-year students to be higher compared with first- to third-year students. In the standing state, there was a trend of the autonomic nervous system activity response of fourth-year students to be higher compared with first- to third-year students. Autonomic responses to psychological stress induced the activation of sympathetic nerves with inhibition of the parasympathetic nervous system (2). In the standing state, there were several cases in the fatigue state with a higher autonomic nervous system activity response (data not shown). The present results suggest that stress may influence autonomic nervous activity in fourth-year nursing students.

Long sleep hours and a regular diet are considered the backbone of a healthy student lifestyle. Sleep decreases sympathetic nerve activity (2). From our previous study (11) it was revealed that fourth-year nursing students had longer studying hours with less sleeping hours. Therefore, the clinical training and study hours had an effect on the life rhythm of fourth-year nursing students. Additionally, lifestyle is affected by stresses, including clinical nursing practice and studying for a national board examination.

The stress response is mediated by the stress system, which is located in the central nervous system and peripheral organs. The main stress system includes the hypothalamic pituitary axis and the systemic sympathetic adrenomedullary systems (4). Stress is a major contributor to psychosocial and physical pathological conditions (4). Chronic stress can cause physical, behavioral and/or neuropsychiatric manifestations, such as anxiety, depression, executive and/or cognitive dysfunction, and sleep disorders such as insomnia (4). Increased levels 
of corticotrophin-releasing hormone and/or stress system abnormalities have been reported in behavioral and neuropsychiatric disorders, such as anxiety, depression and eating disorders. Low corticotrophin-releasing hormone activity has also been implicated in depression (4). All these disorders are associated with dysfunction of the stress system (4).

Studying stress among nursing students is important as this condition is associated with academic performance, which can influence the quality of care in their future professional life. It is necessary to design effective prevention and intervention strategies for mental health problems in nursing students through stress management. Determining how stress occurs among nursing students is an essential step in preventing and reducing the progression of health problems to more severe states. There is widespread agreement that preventing health problems is a better strategy compared to treating them after they become apparent $(12,13)$. Interventions aimed at reducing the risk of health problems by treating autonomic nervous system dysfunction may produce better results, although guidance in effective stress management may be essential.

The limitations of the present study were that it included a relatively small number of participants and that the context was confined to a particular geographic location. However, the study offers certain valuable insights into the autonomic nervous system dysfunction among nursing students.

In conclusion, the present study confirmed that the activity of the autonomic nervous system in the resting state in fourth-year nursing students was low, there was parasympathetic hypotension and there was a trend of the sympathetic nervous system activity of fourth-year students to be higher compared with first- to third-year students. In the standing state, there was a trend of the autonomic nervous system activity response of fourth-year students to be higher compared with first- to third-year students. By correcting stress in fourth-year nursing students, it may be possible to prevent the development of health problems.

\section{Acknowledgements}

The authors express their sincere gratitude to the participants in the study who generously provided their time and trust. The authors would like to express their gratitude to Ms. Chieko Fujii and Mr. Motoi Nagaya for their technical assistance.

\section{References}

1. Hamill RW, Shapiro RE and Vizzard MA: Peripheral autonomic nervous system. In: Primer on the autonomic nervous system. Robertson D, Biaggioni I, Burnstock G,Low PA and Paton JFR (eds). 3rd edition. Academic press, London, UK, pp17-26, 2012.

2. Ziegler MG: Psychological stress and the autonomic nervous system. In: Primer on the autonomic nervous system. Robertson D, Biaggioni I, Burnstock G, Low PA and Paton JFR (eds). 3rd edition. Academic press, London, UK, pp291-293, 2012.

3. Carter C and Tranel D: Mind-Body interactions. In: Primer on the autonomic nervous system. Robertson D, Biaggioni I, Burnstock G, Low PA and Paton JFR (eds). 3rd edition. Academic press, London, UK, pp295-299, 2012.

4. Chrousos GP: Stress and disorders of the stress system. Nat Rev Endocrinol 5: 374-381, 2009.

5. Costa ALS: Stress in nursing students: Construction of determining factors. REME Rev Min Enfem 11: 414-419, 2007.

6. Masamura K, Iwamoto M, Ichihara K, Fujisawa R, Azuma R, Sugiyama S, Kunitsugu I, Okuda M and Hobara T: Relationships between student nurses stress and daily life when engaged in clinical practice. Yamaguchi-igaku 52: 13-21, 2003.

7. Hirose K, Minegishi H, Seto M, Shoda M, Sakata S and Furuya T: College student stress during basic clinical nursing practice: Physical and psychological stress response due to time-serial change. Annual Reports of Gunma Prefectural College of Health Sciences 5: 65-75, 1998.

8. Watson R, Gardiner E, Hogston R, Gibson H, Stimpson A, Wrate R and Deary I: A longitudinal study of stress and psychological distress in nurses and nursing students. J Clin Nurs 18: 270-278, 2009.

9. Timmins F, Corroon AM, Byrne G and Mooney B: The challenge of contemporary nurse education programmes. Perceived stressors of nursing students: Mental health and related lifestyle issues. J Psychiatr Ment Health Nurs 18: 758-766, 2011.

10. Pomeranz B, Macaulay RJ, Caudill MA, Kutz I, Adam D, Gordon D, Kilborn KM, Barger AC, Shannon DC, Cohen RJ, et al: Assessment of autonomic function in humans by heart rate spectral analysis. Am J Physiol 248: H151-H153, 1985.

11. Fukumoto R, Hasegawa M, Takishita Y, Iwawaki Y, Kawaguchi A and Yamanaka R: A study of lifestyle and dietary habits in Japanese nursing students. Sch J Arts Humanit Soc Sci 3 (1B): 139-143, 2015.

12. Garrosa E, Moreno-Jiménez B, Liang Y and González JL: The relationship between socio-demographic variables, job stressors, burnout, and hardy personality in nurses: An exploratory study. Int J Nurs Stud 45: 418-427, 2008.

13. da Silva RM, Goulart CT, Lopes LFD, Serrano PM, Costa ALS and de Azevedo Guido L: Hardy personality and burnout syndrome among nursing students in three Brazilian universities-an analytic study. BMC Nurs 13: 9, 2014. 